\title{
Practical methods for handling human periodontal ligament stem cells in serum-free and serum-containing culture conditions under hypoxia: implications for regenerative medicine
}

\author{
Dai Murabayashi ${ }^{1} \cdot$ Mai Mochizuki $^{1} \cdot$ Yuichi Tamaki $^{1} \cdot$ Taka Nakahara $^{1}$
}

Received: 13 December 2016/ Accepted: 18 January 2017/Published online: 6 February 2017

(C) The Author(s) 2017. This article is published with open access at Springerlink.com

\begin{abstract}
Stem cell-based therapies depend on the reliable expansion of patient-derived mesenchymal stem cells (MSCs) in vitro. The supplementation of cell culture media with serum is associated with several risks; accordingly, serum-free media are commercially available for cell culture. Furthermore, hypoxia is known to accelerate the expansion of MSCs. The present study aimed to characterize the properties of periodontal ligament-derived MSCs (PDLSCs) cultivated in serum-free and serum-containing media, under hypoxic and normoxic conditions. Cell growth, gene and protein expression, cytodifferentiation potential, genomic stability, cytotoxic response, and in vivo hard tissue generation of PDLSCs were examined. Our findings indicated that cultivation in serum-free medium does not affect the MSC phenotype or chromosomal stability of PDLSCs. PDLSCs expanded in serum-free medium exhibited more active growth than in fetal bovine serum-containing medium. We found that hypoxia does not alter the cell growth of PDLSCs under serum-free conditions, but inhibits their osteogenic and adipogenic cytodifferentiation while enabling maintenance of their multidifferentiation potential regardless of the presence of serum. PDLSCs expanded in serum-free medium were found to retain common MSC characteristics, including the
\end{abstract}

Electronic supplementary material The online version of this article (doi:10.1007/s13577-017-0161-2) contains supplementary material, which is available to authorized users.

Taka Nakahara

t.nakahara@tky.ndu.ac.jp

1 Department of Developmental and Regenerative Dentistry, School of Life Dentistry at Tokyo, The Nippon Dental University, 1-9-20 Fujimi, Chiyoda-ku, Tokyo 102-8159, Japan capacity for hard tissue formation in vivo. However, PDLSCs cultured in serum-free culture conditions were more susceptible to damage following exposure to extrinsic cytotoxic stimuli than those cultured in medium supplemented with serum, suggesting that serum-free culture conditions do not exert protective effects against cytotoxicity on PDLSC cultures. The present work provides a comparative evaluation of cell culture in serum-free and serum-containing media, under hypoxic and normoxic conditions, for applications in regenerative medicine.

Keywords Periodontal ligament $\cdot$ Mesenchymal stem cell $\cdot$ Serum-free culture $\cdot$ Hypoxia $\cdot$ Cytotoxic susceptibility

\section{Introduction}

Mesenchymal stem cells (MSCs) comprise a multipotent cell population capable of extensive proliferation and differentiation into a variety of cell types including osteoblasts, adipocytes, chondrocytes, myoblasts, and neurons $[1,2]$. MSCs can be efficiently isolated from several tissues including bone marrow, adipose tissue, and umbilical cord (Wharton's jelly) [3-5]. In addition, teeth, which are usually discarded during common extraction procedures, represent a source of multiple types of dental tissue-derived MSCs [6]. We previously reported the successful isolation and characterization of four types of human dental MSCs derived from dental pulp, periodontal ligament (PDL), apical papilla, and dental follicle, which were all collected from extracted mature or immature wisdom teeth [7]. Notably, these dental MSCs exhibited greater proliferative ability than iliac bone marrow-derived MSCs along with similar multidifferentiation potential and gene/protein 
expression profiles. Furthermore, we and other researchers have reported that PDL-derived MSCs (PDLSCs) in particular are beneficial for stem cell therapy in preclinical trials with large animals [8-10].

In conventional in vitro MSC culture, supplementation of culture media with fetal bovine serum (FBS) is essential for facilitating various cell behaviors such as cell attachment to culture dishes, cell growth, and cytodifferentiation. However, the use of FBS involves potential risks such as xenogeneic immune response and pathogenic infection $[11,12]$; accordingly, several FBS-free culture media for expanding MSCs have been developed for commercial use [13-15]. In addition, low oxygen tension in the cell culture environment has been shown to exert a positive effect on cell growth and proliferation, enabling enhanced numbers of MSCs to be obtained in vitro culture [16, 17]. Hypoxic culture conditions, therefore, represent a potential alternative method for substantially accelerating MSC expansion.

The cellular phenotypes and behaviors of dental MSCs including PDLSCs, such as cell growth, gene/protein expression, cytodifferentiation potential, genomic stability, cytotoxic reactions, and in vivo tissue generation under serum-free and hypoxic culture conditions, remain poorly understood. Here, we aimed to characterize the stem cell properties of PDLSCs under serum-free and hypoxic culture conditions, and to establish practical cell culture methods for obtaining cells for clinical applications and cell-based therapies.

\section{Materials and methods}

\section{Cell isolation and cell culture}

Normal human third molars were obtained from healthy patients aged 27-32 years undergoing tooth extraction. Cell isolation and culture of PDLSCs were performed according to our previous study [7]. Briefly, PDL tissue was gently scraped from the middle third of the tooth root using a sterilized razor blade. The collected tissues were minced and then digested in a solution containing $3 \mathrm{mg} / \mathrm{mL}$ collagenase type I (Merck KGaA, Darmstadt, Germany) and $4 \mathrm{mg} / \mathrm{mL}$ dispase (Wako Pure Chemical Industries, Osaka, Japan) for $1 \mathrm{~h}$ at $37{ }^{\circ} \mathrm{C}$. The cells were passed through a $70-\mu \mathrm{m}$ cell strainer; the resulting single-cell suspensions $\left(1 \times 10^{5}\right.$ cells/dish $)$ were seeded into $100-\mathrm{mm}$ dishes and cultured in Dulbecco's modified Eagle's medium/Ham's nutrient mixture F12 (Thermo Fisher Scientific, Waltham, MA, USA) supplemented with 15\% FBS (Lot No: 027K0361, Merck KGaA), $100 \mu \mathrm{M}$ glutamate (GlutaMAX I; Thermo Fisher Scientific), 0.1\% MEM nonessential amino acids (Thermo Fisher Scientific), $50 \mathrm{U} / \mathrm{mL}$ penicillin, $50 \mu \mathrm{g} / \mathrm{mL}$ streptomycin, and $0.25 \mathrm{mg} / \mathrm{mL}$ amphotericin B (Fungizone). This recipe was designated as FBS-containing medium (FCM). Upon reaching 70-80\% confluence, cells were detached using calcium- and magnesium-free phosphate-buffered saline (PBS) containing $0.25 \%$ trypsin and $0.02 \%$ ethylenediaminetetraacetic acid (EDTA). Then, the detached cells were subcultured at a 1:3 split ratio. The cells were cultured in either FCM or FBS (serum)-free culture medium supplemented with MSC-T4 supplement [18] [Cell Science Technology Institute (CSTI), Sendai, Japan]; this medium was designated as SFM. For SFM cell culture, culture dishes/plates were precoated with MSC-T4 coating reagent (CSTI) [18].

Cell cultures were maintained at $37{ }^{\circ} \mathrm{C}$ in $4.7 \% \mathrm{CO}_{2}$ humidified air, which was designated as the normoxic condition (ambient $\mathrm{O}_{2}$ concentration). For hypoxic cell culture, cells were maintained in the same manner, except that $3 \% \mathrm{O}_{2}$, balanced with $\mathrm{N}_{2}$, was utilized via an $\mathrm{O}_{2}$ - and $\mathrm{CO}_{2}$-controlled multi-gas incubator (MCO-5M; Sanyo, Osaka, Japan). Culture media were changed every 3-4 days. Cells at passage 3 or 4 were used for the subsequent experiments. For each experiment, cells derived from at least three different individuals were used.

\section{Morphological analysis of cultured cells}

The cell morphology of PDLSCs cultured in SFM or FCM was evaluated according to our previous study [7], with minor modifications. Briefly, 3000 cells were randomly selected in subconfluent cultures. Spindle-shaped cells, which were defined as cells that extend a thin process at least three times longer than their cell body, were counted via microscopic observation. The results were expressed as the percentage of spindle-shaped cells with long cell processes.

\section{Cell growth evaluation}

Cells $\left(1 \times 10^{4}\right.$ cells/well $)$ were seeded into 24-multiwell plates and cultured with SFM or FCM under normoxic or hypoxic conditions. Cultured cells were collected by adding trypsin-EDTA and counted in triplicate using a hemocytometer every $48 \mathrm{~h}$ for 14 days. The population doubling time (PDT) was calculated using the following formula: $\left(t-t_{0}\right) \log _{2} / \log \left(N-N_{0}\right)$, where $t$ is time (hours), $N$ is the number of harvested cells, and $N_{0}$ is the number of cells in the inoculum.

\section{Flow cytometry for cell cycle analysis}

Cells collected by the trypsin-EDTA treatment were fixed with cold $70 \%$ ethanol for $2 \mathrm{~h}$ at $-20{ }^{\circ} \mathrm{C}$. After washing 
with PBS, the fixed cells were stained with propidium iodide (PI) for $30 \mathrm{~min}$ at room temperature (RT). The PIelicited fluorescence was measured using a Guava ${ }^{\mathrm{TM}}$ EasyCyte HT instrument and Guava ${ }^{\mathrm{TM}}$ cell cycle software version 2.7 (Guava Technologies, Hayward, CA, USA).

\section{Flow cytometry for cell-surface marker analysis}

After trypsinizing cultured cells, the cells were fixed in cold methanol for $10 \mathrm{~min}$ at $4{ }^{\circ} \mathrm{C}$. Cells were then incubated for $1 \mathrm{~h}$ at RT with an appropriate amount of the following antibodies: fluorescein isothiocyanate (FITC)-conjugated antibodies against CD14, CD90, and CD105 (Becton-Dickinson, San Jose, CA, USA); CD34 and CD44 (Beckman Coulter, Fullerton, CA, USA); and phycoerythrin-conjugated mouse immunoglobulin (Ig) G1 antibody (Becton-Dickinson). The expression profiles were analyzed using a Guava ${ }^{\mathrm{TM}}$ EasyCyte HT and Guava $^{\mathrm{TM}}$ Express Plus (version 2.7) software (Merck $\mathrm{KGaA}$ ).

\section{Reverse-transcription polymerase chain reaction (RT-PCR)}

RT-PCR was performed as previously described [19]. The specific primer sequences and PCR amplification conditions are listed in Table 1.

\section{In vitro multilineage differentiation}

In vitro osteogenic- and adipogenic differentiation experiments in PDLSCs were performed according to our previous study [7]. For chondrogenic differentiation, a pelleted micromass of $1 \times 10^{5}$ cells was formed by centrifugation at $430 \times g$ for $5 \mathrm{~min}$ and then cultured with $\alpha$ MEM containing $10 \%$ FBS, $10 \mathrm{ng} / \mathrm{mL}$ transforming growth factor- $\beta 1$ (PeproTech, Rocky Hill, NJ, USA), $50 \mathrm{mM}{ }_{\mathrm{L}}$-ascorbic acid 2-phosphate magnesium salt $n$ hydrate, and $50 \mathrm{mM}$ ITS +1 (Merck KGaA) for 4 weeks. After the differentiation, pellets were fixed with $10 \%$ neutral buffered formalin, embedded in paraffin, and cut into sections of $5-\mu \mathrm{m}$ thickness for histological analysis. Chondrogenic differentiation was evaluated by staining with solutions of $1 \%$ Alcian blue and $0.1 \%$ Safranin O, and by immunohistochemistry using a rabbit polyclonal antitype-II collagen antibody (1:50; Santa Cruz Biotechnology, Dallas, TX, USA).

\section{Immunocytofluorescence}

Cultured cells were plated at $1 \times 10^{5}$ cells/well in four-well chamber slides and fixed with cold methanol for $10 \mathrm{~min}$ at $-30{ }^{\circ} \mathrm{C}$. After washing with PBS, cells were incubated in Blocking One Histo (Nacalai Tesque, Kyoto, Japan) for $10 \mathrm{~min}$ at RT. Cultures were incubated with the following

Table 1 Primer sequences and amplification conditions for RT-PCR analysis

\begin{tabular}{|c|c|c|c|c|c|}
\hline Gene & Primer sequences, $5^{\prime}$ to $3^{\prime}$ & $\begin{array}{l}\text { Product size } \\
\text { (bp) }\end{array}$ & $\begin{array}{l}\text { Annealing } \\
\text { temp. }\left({ }^{\circ} \mathrm{C}\right)\end{array}$ & $\begin{array}{l}\text { PCR } \\
\text { cycles }\end{array}$ & $\begin{array}{l}\text { GenBank } \\
\text { accession number }\end{array}$ \\
\hline Vimentin & $\begin{array}{l}\text { F: GGGACCTCTACGAGGAGGAG } \\
\text { R: CGCATTGTCAACATCCTGTC }\end{array}$ & 200 & 55 & 35 & NM_003380 \\
\hline Collagen I & $\begin{array}{l}\text { F: CCAAATCTGTCTCCCCAGAA } \\
\text { R: TCAAAAACGAAGGGGAGATG }\end{array}$ & 214 & 55 & 35 & NM_000088 \\
\hline Runx2 & $\begin{array}{l}\text { F: CCCCACGACAACCGCACCAT } \\
\text { R: GTCCACTCCGGCCCACAAATC }\end{array}$ & 292 & 59 & 35 & NM_001015051 \\
\hline Nestin & $\begin{array}{l}\text { F: AACAGCGACGGAGGTCTCTA } \\
\text { R: TTCTCTTGTCCCGCAGACTT }\end{array}$ & 220 & 55 & 35 & NM_006617 \\
\hline Nanog & $\begin{array}{l}\text { F: ACCTTCCAATGTGGAGCAAC } \\
\text { R: GAATTTGGCTGGAACTGCAT }\end{array}$ & 199 & 55 & 35 & NM_024865 \\
\hline Oct $3 / 4$ & $\begin{array}{l}\text { F: GACAGGGGGAGGGGAGGAGCTAGG } \\
\text { R: CTTCCCTCCAACCAGTTGCCCCAAAC }\end{array}$ & 144 & 60 & 35 & NM_001173531 \\
\hline Sox 2 & $\begin{array}{l}\text { F: AACCCCAAGATGCACAACTC } \\
\text { R: CGGGGCCGGTATTTATAATC }\end{array}$ & 152 & 60 & 40 & NM_003106 \\
\hline GAPDH & $\begin{array}{l}\text { F: GAGTCAACGGATTTGGTCGT } \\
\text { R: TTGATTTTGGAGGGATCTCG }\end{array}$ & 238 & 55 & 35 & NM_002046 \\
\hline
\end{tabular}

Collagen I Collagen type I alpha 1 chain, Runx2 Runt-related transcription factor 2, Nanog Nanog homeobox, Oct3/4 POU class 5 homeobox 1 (POU5F1), Sox2 SRY-box 2, GAPDH glyceraldehyde-3-phosphate dehydrogenase 
primary antibodies at $4{ }^{\circ} \mathrm{C}$ overnight: rabbit polyclonal antinestin, rabbit polyclonal anti-neurofilament-200, and chicken polyclonal anti-ßIII-tubulin (all diluted 1:1000; Merck KGaA). After washing with PBS, the samples were incubated with the following secondary antibodies for $30 \mathrm{~min}$ at RT in the dark: Alexa Fluor 488-conjugated goat anti-mouse IgG, Alexa Fluor 488-conjugated donkey antirabbit IgG, and Alexa Fluor 488-conjugated goat antichicken IgY (all diluted 1:1000; Thermo Fisher Scientific). The samples were washed with PBS three times for $5 \mathrm{~min}$ each and mounted with Vectashield mounting medium containing 4',6-diamidino-2-phenylindole (Vector Laboratories, Burlingame, CA, USA). For negative controls, the primary antibody was omitted during immunostaining. Images were obtained using a Biorevo BZ-9000 microscope (Keyence, Osaka, Japan).

\section{Karyotype analysis}

Karyotype G-band analysis was performed as previously described [20].

\section{Assessment of cytotoxic stimuli-induced cell viability and cell damage}

To investigate whether SFM or FCM cultures are affected by extrinsic cytotoxicity, cultured PDLSCs were subjected to treatment with extrinsic stimuli via exposure to staurosporine, hydrogen peroxide $\left(\mathrm{H}_{2} \mathrm{O}_{2}\right)$, or ultraviolet radiation (UV), and cellular stress/damage was detected. Cells were seeded into 24 -multiwell plates $\left(1 \times 10^{5}\right.$ cells/well $)$ and treated with the following: $1 \mu \mathrm{M}$ staurosporine (Wako Pure Chemical Industries) or $1 \mu \mathrm{M} \mathrm{H}_{2} \mathrm{O}_{2}$ (Wako Pure Chemical Industries) for $12 \mathrm{~h}$ at $37^{\circ} \mathrm{C}$. Alternatively, cells were seeded into $60-\mathrm{mm}$ dishes $\left(1 \times 10^{6}\right.$ cells/dish $)$ and irradiated with UV light using an UV transilluminator (UVP, Upland, CA) for $30 \mathrm{~min}$ at RT.

\section{Detection of cell viability using an 3-(4,5- dimethylthiazol-2-yl)-2,5-diphenyltetrazolium bromide (MTT) assay}

Cell viability of the PDL-derived cells exposed to cytotoxic stimuli was assessed via the MTT colorimetric assay. Following treatment with staurosporine, $\mathrm{H}_{2} \mathrm{O}_{2}$, or UV, $50 \mu \mathrm{L}$ MTT-tetrazolium salts $(5 \mathrm{mg} / \mathrm{mL})$ (Merck KGaA) in PBS was added to each well in 24-multiwell plates. After $2 \mathrm{~h}$ incubation at $37{ }^{\circ} \mathrm{C}$, the formazan crystals were dissolved by adding $300 \mu \mathrm{L}$ isopropanol. Absorbance of the supernatant fluid was measured at $570 \mathrm{~nm}$ wavelength using a Corona Grating Microplate Reader (Hitachi High-Technologies). The cell viability rate $(\%)$ was calculated as the sample optical density $(\mathrm{OD})$ divided by control OD $\times 100$.
Detection of cellular stress/damage by flow cytometric analysis with Annexin V/PI staining

Cellular stress/damage in the aforementioned cytotoxic stimuli-treated PDLSCs was determined by flow cytometric analysis using an Annexin V-FITC/PI system (Guava Technologies), according to the manufacturer's instructions. Briefly, cells exposed to each stimulus were incubated with $100 \mu \mathrm{L}$ Guava Nexin Reagent for 20 min at RT in the dark and analyzed using the Guava ${ }^{\mathrm{TM}}$ EasyCyte HT and Guava ${ }^{\mathrm{TM}}$ InCyte System (version 2.7). Annexin V-positivity and PI-negativity indicated the presence of early apoptotic cells, and Annexin V- and PI-double positivity indicated the presence of late apoptotic cells. In this study, both classes of fractionated cells were considered to represent damaged cells. The damaged cell rate (\%) was calculated as the number of damaged cells divided by the number of total cells tested $\times 100$.

\section{In vivo transplantation and histological evaluation}

To examine the hard tissue formation capacity in vivo, PDLSCs expanded using SFM or FCM were subcutaneously transplanted under the dorsal skin of 6-week-old female nude mice (BALB/C-nu/nu; Nihon Clea, Tokyo, Japan). The cell/scaffold construct consisted of a mixture of approximately $1 \times 10^{6}$ cells and $400 \mu \mathrm{L}$ collagen gel (Nitta Gelatin, Osaka, Japan) with $40 \mathrm{mg}$ hydroxyapatite (HA)/ $\beta$ TCP-scaffold (Ceraform ${ }^{\circledR}$; NGK Spark Plug, Aichi, Japan). All transplants were collected for histological analysis at 16 weeks post-transplantation ( $n=6$ per group).

Histological and immunohistochemical examinations were performed as previously described [21]. Primary antibodies were used as follows: mouse monoclonal antihuman-specific vimentin (1:10,000; Merck KGaA), rabbit polyclonal anti-osteopontin (OPN; 1:1000; Abcam, Cambridge, MA, USA), and rabbit polyclonal anti-cementum attachment protein (CAP; 1:100; LifeSpan Biosciences, Seattle, WA, USA). For negative controls, the primary antibody was omitted during immunostaining.

\section{Statistical analysis}

Quantitative data were obtained from three independent experiments. All samples were analyzed in triplicate and the data were expressed as the mean \pm standard deviation. Representative results were indicated in each experiment. Statistical analysis was performed using two-way ANOVA with Bonferroni's correction or Fisher's Exact test. A probability of $P<0.01$ was considered to indicate a statistically significant difference. Data were analyzed using IBM SPSS Statistics software (version 23.0; IBM Japan, Tokyo, Japan). 
a

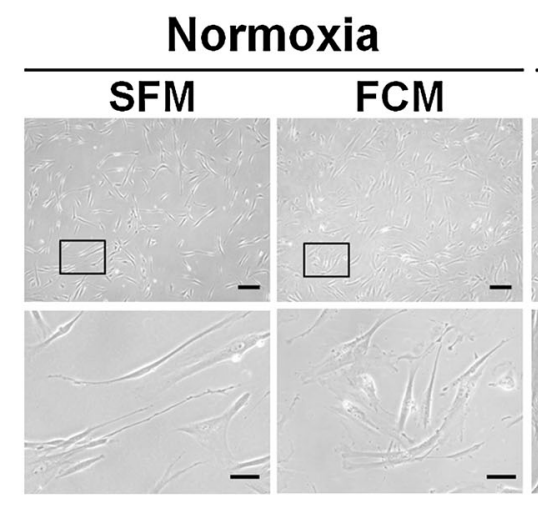

C

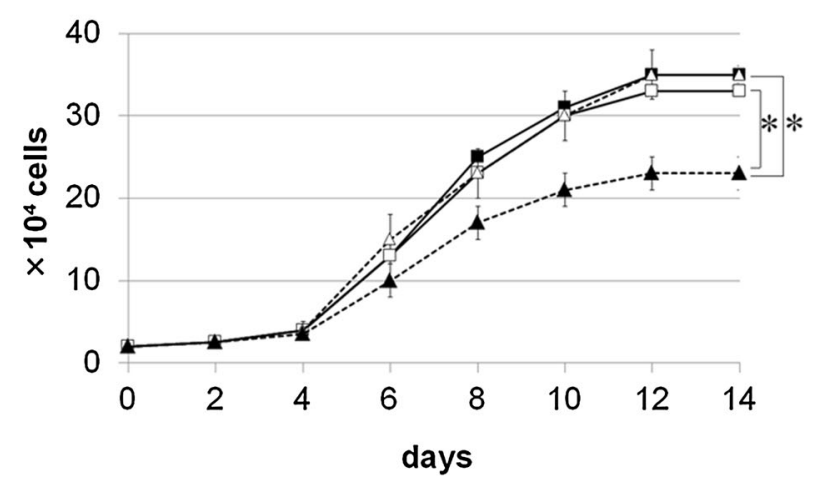

$\rightarrow-\mathrm{SFM}$ in Normo $-\triangle-\mathrm{SFM}$ in $3 \% \mathrm{O}_{2}$ $-\mathrm{A}$ FCM in Normo $-\square-\mathrm{FCM}$ in $3 \% \mathrm{O}_{2}$

Fig. 1 Morphological evaluation and cell growth characteristics of PDLSCs cultured in the presence or absence of FBS, under either normoxic or hypoxic culture conditions. a Phase-contrast images of PDLSCs cultured in serum-free medium (SFM) or FBS-containing medium $(\mathrm{FCM})$ under normoxic (normo) or hypoxic $\left(3 \% \mathrm{O}_{2}\right)$ culture conditions; the lower panels show enlarged views indicated by the boxed area in the upper panels. All the PDLSC cultures primarily showed fibroblastic cell morphology when cultivated in SFM and FCM under normoxic and hypoxic conditions. In particular, SFMcultured PDLSCs exhibited a more spindle-shaped morphology under hypoxic conditions. Scale bars $200 \mu \mathrm{m}$ (upper panels); $50 \mu \mathrm{m}$ (lower panels). $\mathbf{b}$ Morphological evaluation of the length of cell processes of PDLSCs cultured in SFM or FCM based on hypoxia $(* P<0.01)$. The cell rate $(\%)$ indicates the number of cells with longer processes divided by the number of total cells examined. c Growth curve analysis of SFM- and FCM-cultured PDLSCs under hypoxic and normoxic conditions at 14 days post-seeding; FCM-cells under

\section{Results and discussion}

\section{PDLSCs cultured in SFM exhibit more active cell growth than those cultured in FCM under normoxic culture conditions}

SFM- and FCM-cultured PDLSCs showed typical fibroblastic cell morphology under normoxic culture conditions
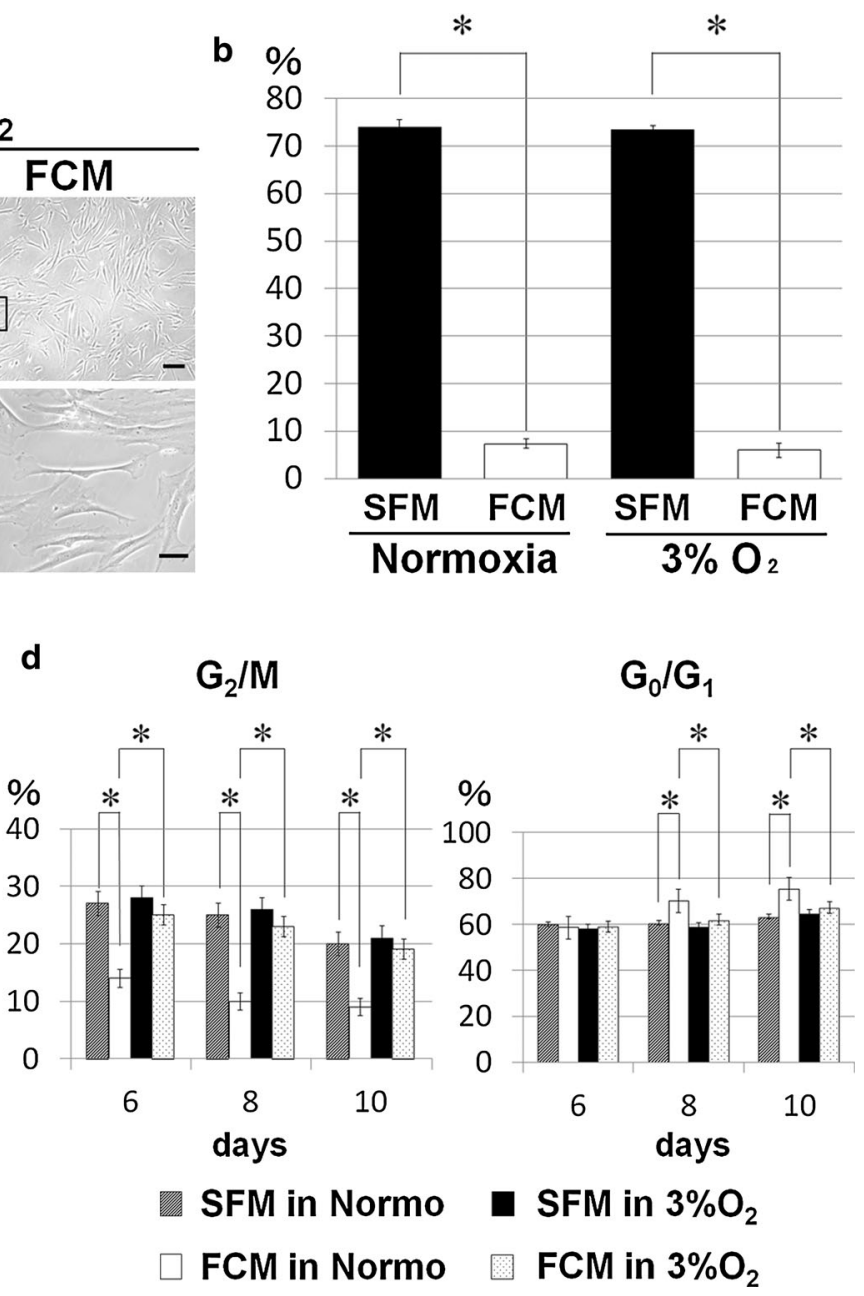

hypoxia (open squares) and SFM-cells under normoxia (closed squares) showed a significantly greater growth rate than FCM-cells under normoxia (closed triangles) at day 8 or later in culture $\left({ }^{*} P<0.01\right)$. Notably, no significant difference was found between the growth of SFM cells under normoxic (closed squares) and hypoxic (open triangles) culture conditions. Further, there was no significant difference in cell growth between SFM cells (open triangles) and FCM cells (open squares) under hypoxic conditions during the culture period. d Flow cytometric analysis for cell cycle status indicated that a greater cell growth of FCM cells under hypoxia and SFM cells under normoxia than FCM cells under normoxia, and that the proportion of cells in G2/M phase was significantly higher, and those in the G0/G1 phase significantly lower, among FCM cells under hypoxia and SFM cells under normoxia relative to FCM cells under normoxia during the logarithmic growth phase in culture period $(* P<0.01)$

(Fig. 1a) although SFM-cultured cells exhibited significantly greater spindle-shaped morphology, with longer cell processes, than FCM-cultured cells (Fig. 1b). Similar findings are described in some previous reports of bone marrow-derived MSCs cultured under serum-free culture conditions $[14,22]$. The cell growth of SFM-cultured PDLSCs under normoxic conditions was significantly higher than that of FCM-cultured cells at day 8 or later in 
culture (Fig. 1c). Flow cytometric analysis of cell cycle status during the logarithmic growth phase also indicated active SFM cell division (Fig. 1d), consistent with previous reports [15, 23].

To examine whether serum-free culture conditions enable the maintenance of stem cell characteristics and normal chromosomal stability under normoxia, phenotype, trilineage differentiation, and karyotype analyses were performed. In addition to the osteogenic/cementogenic, adipogenic, and chondrogenic lineages obtained by trilineage differentiation (Fig. 2a), a common MSC phenotype was indicated by flow cytometry, RT-PCR, and immunofluorescence (Supplemental Fig. 1). PDLSCs cultured in SFM or FCM exhibited normal karyotypes and diploid sets of chromosomes $(2 n=46$; data not shown). Collectively, these findings suggest that the serum-free culture environment does not affect PDLSC chromosomal stability, MSC phenotype, or multidifferentiation potential.

\section{Hypoxia does not alter the cell growth of PDLSCs cultured in SFM}

Hypoxia facilitates the growth of cultured cells under conventional cultivation conditions in the presence of FBS $[16,17]$. Therefore, we investigated whether hypoxia induced similar effects on PDLSC proliferation during cultivation in SFM. Hypoxia did not impact the fibroblastic cell morphology of PDLSCs cultured in SFM or FCM (Fig. 1a) including the significantly longer cell process length in SFM cells (Fig. 1b). However, hypoxia induced the active growth of FCM-PDLSCs as expected but not SFM-cultured PDLSCs, enhancing the proliferation of the former to levels comparable to those of SFM cells cultured under either $\mathrm{O}_{2}$ tension condition (Fig. 1c, d). Similar findings were observed by assessing PDT values, which were shorter $(22.8,22.5$, and $22.7 \mathrm{~h})$ for PDLSCs cultured in SFM under normoxia or hypoxia, and FCM under hypoxia, respectively, than for cells cultured in FCM under normoxia (30.9 h). Furthermore, the common MSC phenotype was observed following trilineage differentiation of SFM- and FCM-cultured PDLSCs (Supplemental Fig. 2 and the following section), suggesting that the hypoxic culture environment does not affect the MSC phenotype of these cells. Taken together, these findings suggest that hypoxic conditions are beneficial for ex vivo expansion of PDLSCs and maintenance of the stem cell phenotype during cultivation in FCM; however, hypoxia is not useful to accelerate the proliferation level of PDLSCs cultured in SFM.
Fig. 2 In vitro multilineage differentiation potential of PDLSCs cultured in the presence or absence of FBS under hypoxic or normoxic culture conditions. a Alizarin red S (ALZ)- and oil red O (ORO)-staining indicate mineralized deposits and lipid clusters, respectively, in SFM- or FCM-cells under normoxic conditions 4 weeks after culturing in the respective cytodifferentiation medium. In contrast, no positive staining was observed in either SFMor FCM-cells under hypoxic conditions $\left(3 \% \mathrm{O}_{2}\right)$. Chondrogenic differentiation was achieved in all the PDLSC-pellet cultures after 4 weeks (Safranin O and Alcian blue); particularly, cytodifferentiation was enhanced under hypoxic culture conditions, as indicated by the intense immunoreactivity for type II collagen (Collagen II); scale bars $50 \mu \mathrm{m}$. b SFM and FCM cells also achieved osteogenic and adipogenic cytodifferentiation after 2 weeks of normoxic cultivation (Normo $2 \mathrm{w}$ ), but failed to exhibit cytodifferentiation into either lineage after 2 weeks of hypoxic cultivation $\left(3 \% \mathrm{O}_{2} 2 \mathrm{w}\right)$. Notably, switching the culture condition from hypoxia for 2 weeks to normoxia for 2 weeks resulted in the development of ALZ-positive mineralized nodules and ORO-positive lipid droplets in SFM and FCM cultures, respectively $\left(3 \% \mathrm{O}_{2} 2 \mathrm{w} \rightarrow\right.$ Normo $\left.2 \mathrm{w}\right)$. c Reverse-transcription polymerase chain reaction analysis revealed that 2-week-hypoxiacultured PDLSCs that failed to undergo osteogenic (Os) and adipogenic (Ad) lineage differentiation exhibited higher expression of the stemness marker genes Oct $3 / 4$, Nanog, and Sox $2\left(3 \% \mathrm{O}_{2}\right)$; after switching to normoxia, PDLSCs lost, or showed a lower expression of, stemness marker genes during cultivation for differentiation into both lineages (Normo)

\section{Hypoxia inhibits osteogenic and adipogenic cytodifferentiation, but maintains the multidifferentiation potential of PDLSCs}

The cytodifferentiation of MSCs under conditions of various low- $\mathrm{O}_{2}$ tension (less than $8 \% \mathrm{O}_{2}$ ) remains elusive. Moreover, successful MSC cytodifferentiation is dependent on the species, cell source, and cell isolation method [24-27]. We next examined the effect of hypoxia on the multidifferentiation potential of PDLSCs. Following culture in the respective induction media for differentiation into osteogenic/cementogenic, adipogenic, or chondrogenic lineages under hypoxic conditions for 4 weeks, no mineralized nodules or lipid vacuoles were observed in cultures maintained using SFM or FCM (Fig. 2a). This suggests that hypoxia inhibits PDLSC osteogenic and adipogenic cytodifferentiation.

To investigate whether PDLSCs cultured in hypoxia maintain their immature state and multidifferentiation potential, we firstly confirmed that osteogenic or adipogenic differentiation could be established under normoxic but not hypoxic conditions for 2 weeks (Fig. 2b). Notably, subsequently switching PDLSCs from hypoxia for 2 weeks to normoxia for 2 weeks allowed successful cytodifferentiation into both osteogenic and adipogenic lineages, as indicated by Alizarin red S-positive- and oil red O-positive-staining, respectively (Fig. 2b). 
a

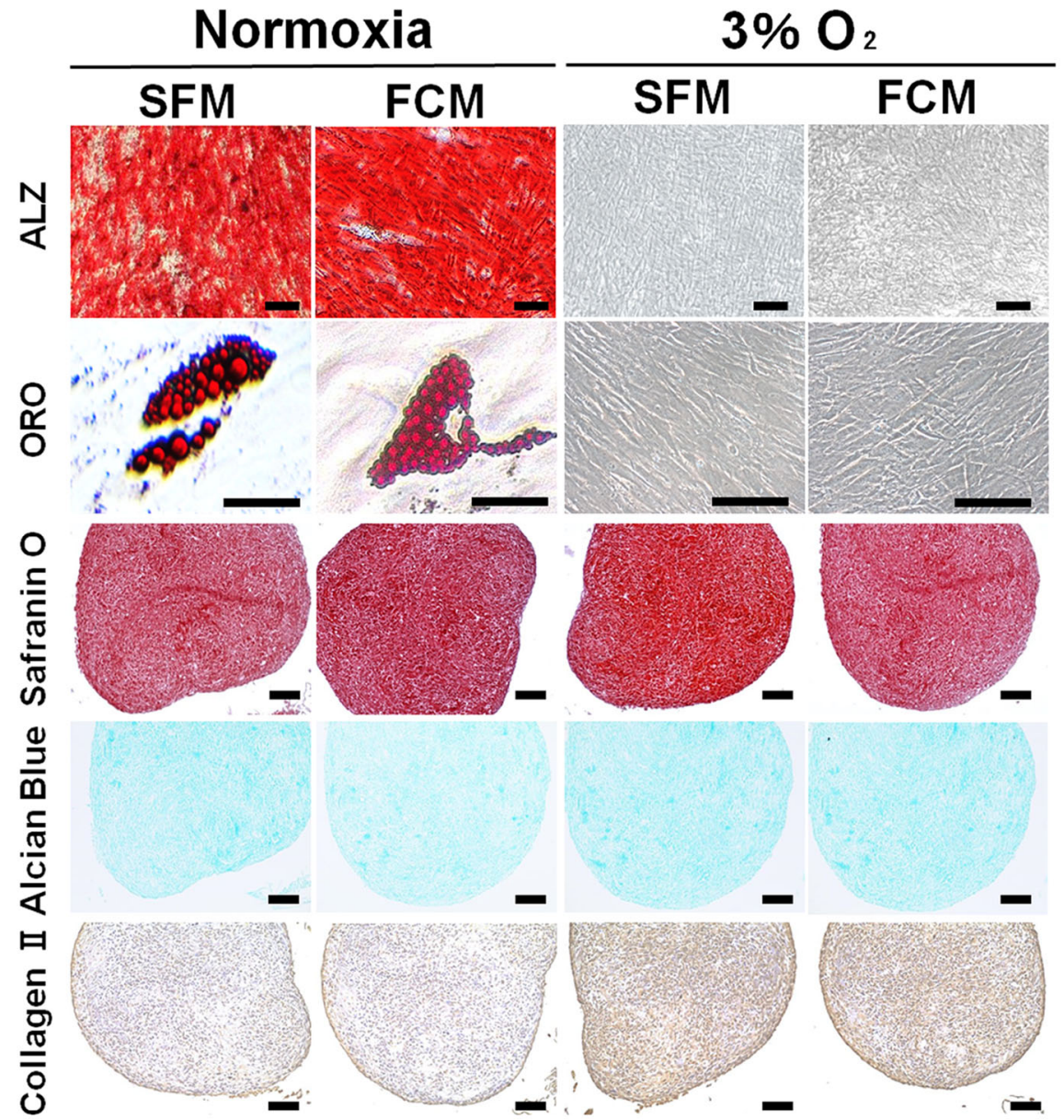

b

C

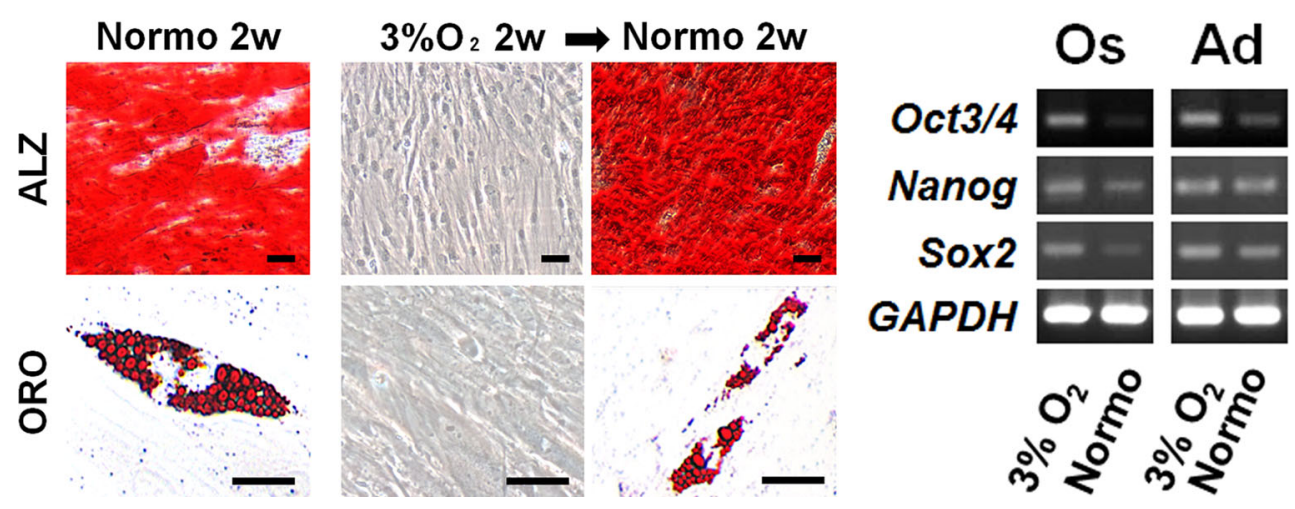

Furthermore, the expression of stemness marker-encoding genes including Oct3/4, Nanog, and Sox2, which are also involved in the maintenance of stemness [28, 29], was higher in the cytodifferentiation-inhibited PDLSCs cultured under hypoxia for 2 weeks than in the cytodifferentiated PDLSCs cultured under normoxia as ascer- tained by RT-PCR (Fig. 2c). This suggests that the former cells maintained their immature state with multidifferentiation potential. In contrast, hypoxia enhanced the chondrogenic differentiation of PDLSCs cultured in SFM or FCM (Fig. 2a), consistent with previous reports [30, 31]. Collectively, our data indicate that hypoxia does not affect 
PDLSC stemness, i.e., multidifferentiation potential, but enhances their chondrogenic differentiation, irrespective of serum use.

\section{PDLSCs are more susceptible to damage by extrinsic cytotoxic stimuli in SFM compared with FCM}

We next determined whether the presence of serum affects cell viability and cellular damage in PDLSCs exposed to various extrinsic stimuli. Cells were treated with widely known cytotoxic stimuli including $\mathrm{H}_{2} \mathrm{O}_{2}[32,33]$, a reactive oxygen species that induces oxidative stress; UV radiation $[34,35]$; or staurosporine $[36,37]$, which typically induces apoptotic death. Exposure to these agents caused degenerative morphologic changes in SFM- and FCM-cultured PDLSCs (Fig. 3a). Cell viability, as indicated by MTT assay, was significantly higher in FCM than in SFM cells (Fig. 3b) whereas the cellular stress/damage, as indicated by flow cytometry with Annexin V-FITC/PI staining, was significantly higher in SFM cells (Fig. 3c, d). Thus, the serum-free culture condition does not appear to exert protective effects against cytotoxicity on cultured cells exposed to extrinsic cytotoxic stimuli such as UV and chemical inducers of apoptosis.

\section{Ex vivo-expanded PDLSCs cultured in SFM generate cementum-like hard tissue in vivo}

We next tested in vivo tissue formation by PDLSCs expanded using SFM or FCM, as shown in Fig. 4. Ex vivoexpanded PDLSC/HA-scaffold constructs were transplanted into the dorsal subcutaneous tissue of immunodeficient mice. Histological examination by hematoxylin and eosin and Masson's trichrome staining showed that SFMor FCM-cultured PDLSC/scaffold constructs but not HA scaffold alone (without cells) generated cementum-like hard tissue at 16 weeks post-implantation. Human-specific vimentin-positive cells, indicative of transplanted PDLSCs, were observed in connective tissue around the de novo hard tissue in both cell/scaffold constructs. Moreover, positive immunoreactivity for OPN, a non-collagenous protein present in mineralized tissues including the cementum [38], and CAP, a collagenous protein that promotes the attachment of mesenchymal stromal cells including cementoblasts [39], was observed within the newly formed hard tissue matrix but not within control transplant-associated fibroblasts and fibrous tissue. These findings indicate that
Fig. 3 Cellular viability and stress/damage induced by extrinsic cytotoxicity under serum-free or FBS-containing culture condition. a Morphological alteration of the serum-free (SFM)- and FBScontaining (FCM)-cultured PDLSCs before (Control) and after cytotoxic treatments with staurosporine (ST), hydrogen peroxide $\left(\mathrm{H}_{2} \mathrm{O}_{2}\right)$, and ultraviolet radiation (UV). All the cytotoxic stimulitreated SFM cells showed damaged appearance with degenerative changes relative to the treated FCM cells; scale bars $100 \mu \mathrm{m}$. b The MTT assay revealed that viability of FCM cells was significantly higher than that of SFM cells after the cytotoxic treatments $(* P<0.01)$. c Flow cytometric analysis by Annexin V-FITC/PI staining showed that a greater amount of damaged cells, which were fractionated as both Annexin V-positive/PI-negative (early apoptotic cells) and Annexin V/PI-double positive (late apoptotic cells), were detected in SFM cultures compared with FCM cultures, following cytotoxic treatment. d Quantification of the flow cytometric analysis for damaged cells $(* P<0.01)$

PDLSCs expanded in SFM ex vivo maintained the capacity for de novo cementogenesis in vivo.

\section{Conclusions and perspectives}

The present study indicates that commercially available SFM may facilitate the greater expansion of PDLSCs than conventional FCM. Moreover, SFM-expanded PDLSCs retained common MSC characteristics including the capacity for in vivo hard tissue formation. We further demonstrated that (1) hypoxia does not alter PDLSC growth under serum-free conditions, (2) hypoxia inhibits PDLSC osteogenic and adipogenic cytodifferentiation but enables maintenance of their multidifferentiation potential regardless of serum content, and (3) PDLSCs are likely damaged by a wide range of extrinsic cytotoxic stimuli under serum-free culture conditions.

Methods for the reliable and rapid expansion of primary cells from patients are necessary to acquire sufficient number of cells ex vivo for cell-based therapies. Commercially available SFM represents an optimal choice to facilitate cell growth but leaves cells susceptible to cell stress/damage induced by exposure to potentially cytotoxic stimuli during routine cell culture protocols. Alternatively, serum supplementation provides cytoprotective effects and enables reliable cell growth, which may be enhanced by hypoxic culture conditions to levels comparable to that in serum-free culture. Conversely, the hypoxic protocol is not necessary for MSC expansion in serum-free culture or for cytodifferentiation induction culture into osteogenic and adipogenic, but not chondrogenic lineages. Collectively, the present findings provide an evaluation of the practical 

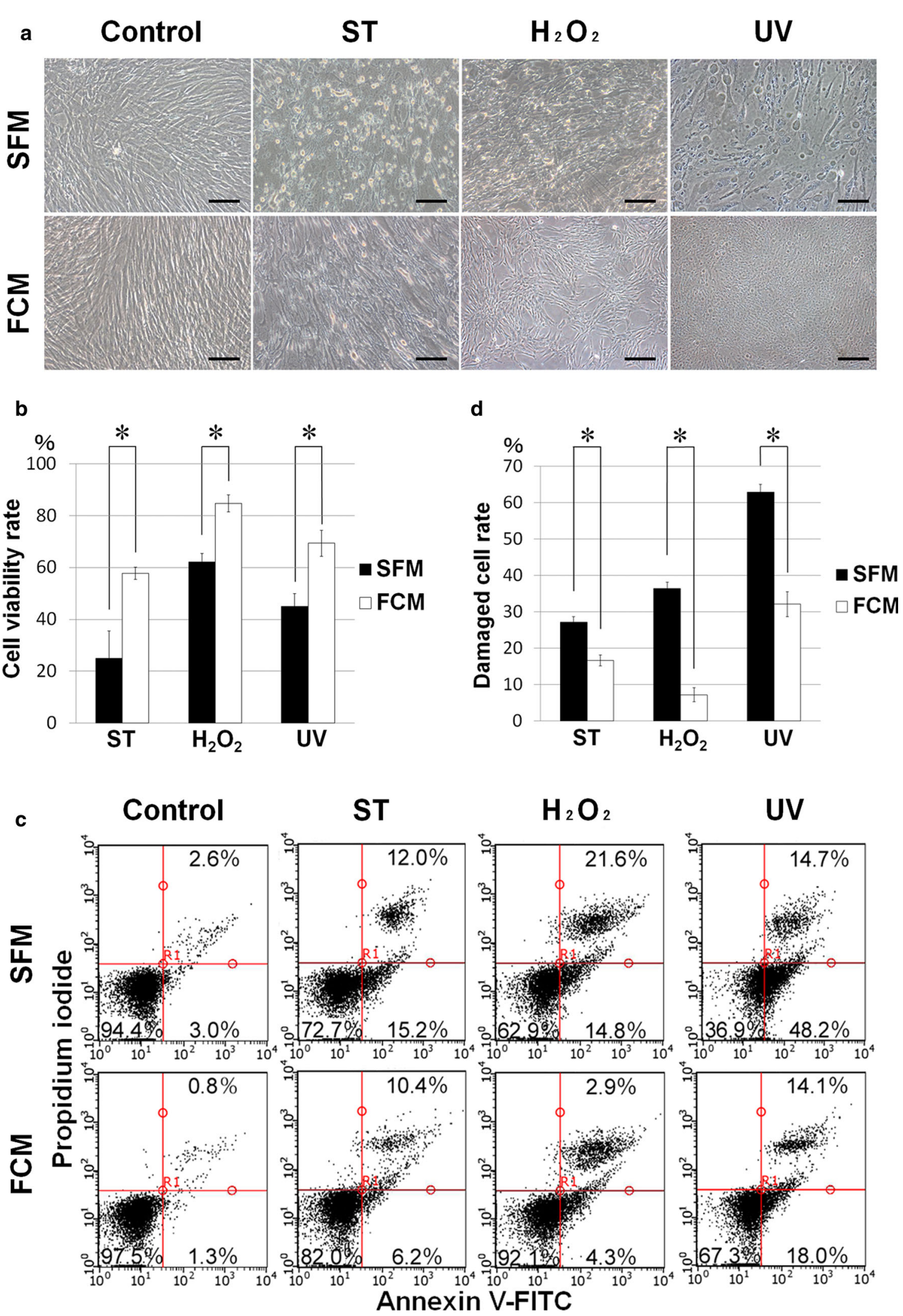
Fig. 4 In vivo transplantation of ex vivo-expanded PDLSCs using serum-free- or FBScontaining medium; histological and immunohistochemical evaluation of cell/ hydroxyapatite (HA)-scaffold constructs containing PDLSCs cultured in serum-free (SFM) or FBS-containing (FCM) medium in nude mice after subcutaneous transplantation. Hematoxylin and eosin (HE) and Masson's trichrome (MT) staining indicated that the newly formed cementum hard tissue, which is indicated by the dashed line, was observed at the surface of HA-scaffold carrier (HA) in the SFM- and FCM-cell constructs. Immunoreactivity for the human-specific vimentin antibody was detected in the fibroblasts in connective tissue around the de novo hard tissue in both types of cell/scaffold constructs. Moreover, the hard tissue matrix was positively immunostained with antibodies against osteopontin (OPN) and cementum attachment protein (CAP), which are typical markers for cementum hard tissue. Neither hard tissue formation nor immunoreactivity for any antibodies was observed in the HA-scaffold alone (Control). The primary antibody was omitted during immunostaining (Negative); scale bars $100 \mu \mathrm{m}$
SFM

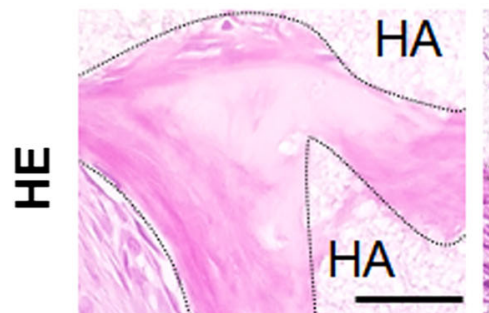

FCM

Control
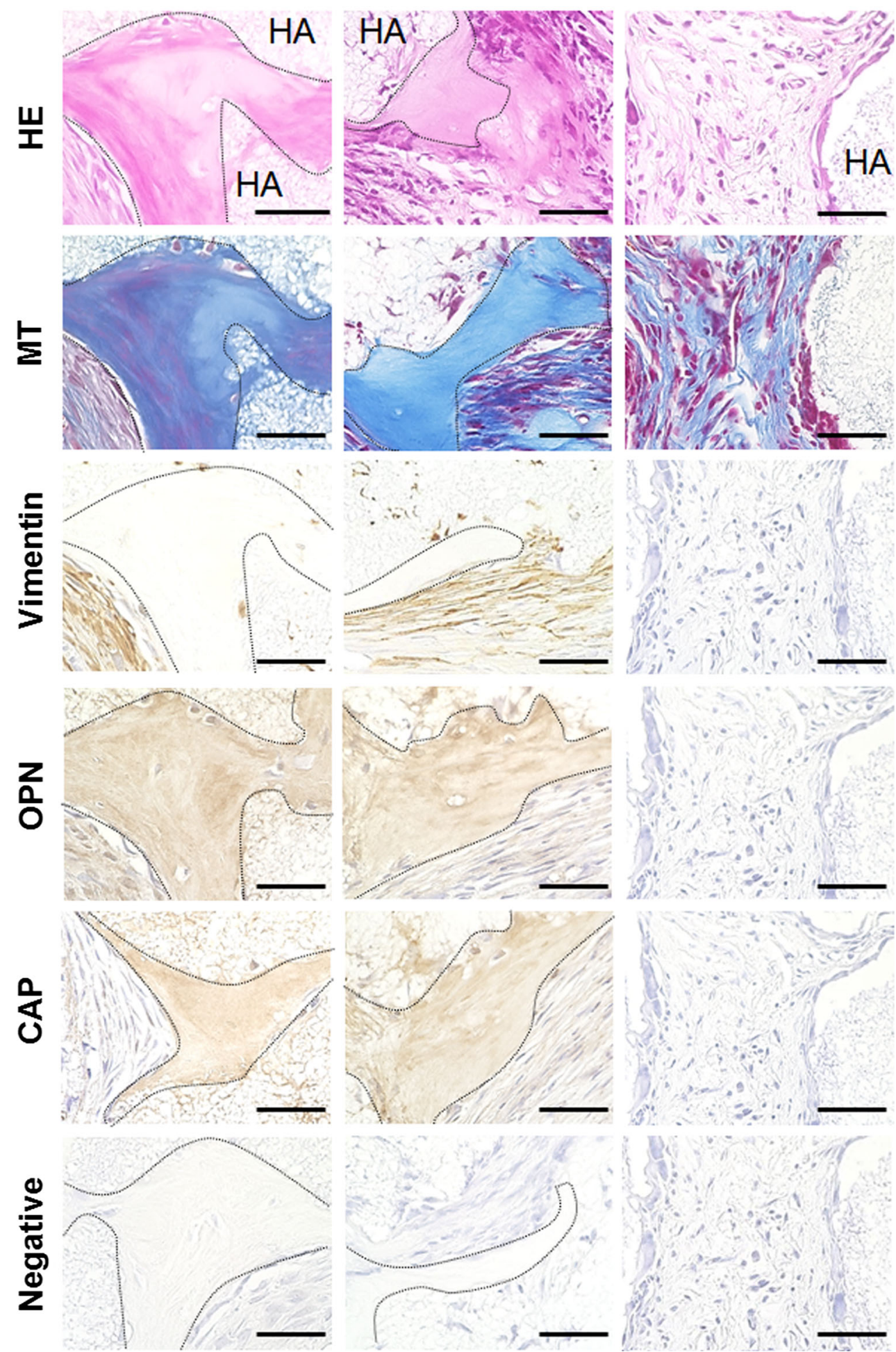

HA
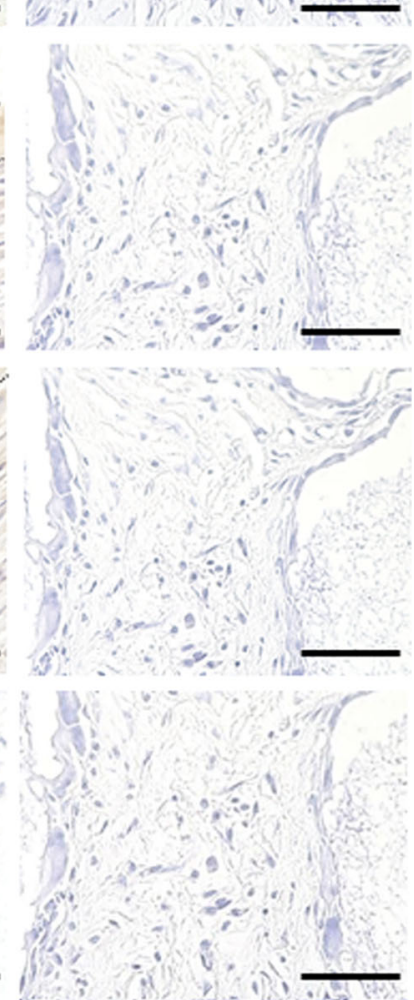

feasibility of serum-free or serum-containing culture conditions for handling PDLSC cultures for clinical applications in regenerative medicine.

Acknowledgements The authors thank Junko Toyomura, Tetsuro Horie, and Kazutoshi Sato for providing technical support and scientific advice. This work was supported by a Grant-in-Aid for Young Scientists (A) (No. 24689073 to T.N.) and a Grant-in-Aid for Scientific Research (B) (No. 15H05046 to T.N.) from the Japan Society for the Promotion of Science (JSPS KAKENHI); and was supported in part by a Research Grant (2013-2014 to T.N.) from the Nippon Dental University. 


\section{Compliance with ethical standards}

Conflict of interest The authors declare that they have no conflicts of interest.

Ethical approval All procedures performed in experiments involving human participants were conducted in accordance with the ethical standards of the ethics committee of Nippon Dental University School of Life Dentistry at Tokyo as well as the 1964 Helsinki declaration and its later amendments, or comparable ethical standards. Informed consent was obtained from all individual participants included in the study. All procedures performed in experiments involving animals were conducted in accordance with the "Regulations for Animal Experimentation and Laboratory Animal Facility" of the Nippon Dental University School of Life Dentistry at Tokyo.

Open Access This article is distributed under the terms of the Creative Commons Attribution 4.0 International License (http://crea tivecommons.org/licenses/by/4.0/), which permits unrestricted use, distribution, and reproduction in any medium, provided you give appropriate credit to the original author(s) and the source, provide a link to the Creative Commons license, and indicate if changes were made.

\section{References}

1. Alhadlaq A, Mao JJ. Mesenchymal stem cells: isolation and therapeutics. Stem Cells Dev. 2004;13:436-48.

2. Huang GT, Gronthos S, Shi S. Mesenchymal stem cells derived from dental tissues vs. those from other sources: their biology and role in regenerative medicine. J Dent Res. 2009;88:792-806.

3. Pittenger MF, Mackay AM, Beck SC, et al. Multilineage potential of adult human mesenchymal stem cells. Science. 1999;284: 143-7.

4. Zuk PA, Zhu M, Ashjian P, et al. Human adipose tissue is a source of multipotent stem cells. Mol Biol Cell. 2002;13: 4279-95.

5. Wang HS, Hung SC, Peng ST, et al. Mesenchymal stem cells in the Wharton's jelly of the human umbilical cord. Stem Cells. 2004;22:1330-7.

6. Seo BM, Miura M, Gronthos S, et al. Investigation of multipotent postnatal stem cells from human periodontal ligament. Lancet. 2004;364:149-55.

7. Tamaki Y, Nakahara T, Ishikawa $H$, Sato S. In vitro analysis of mesenchymal stem cells derived from human teeth and bone marrow. Odontology. 2013;101:121-32.

8. Nakahara T, Nakamura T, Kobayashi E, et al. In situ tissue engineering of periodontal tissues by seeding with periodontal ligament-derived cells. Tissue Eng. 2004;10:537-44.

9. Liu Y, Zheng Y, Ding G, et al. Periodontal ligament stem cellmediated treatment for periodontitis in miniature swine. Stem Cells. 2008;26:1065-73.

10. Washio K, Iwata T, Mizutani M, et al. Assessment of cell sheets derived from human periodontal ligament cells: a pre-clinical study. Cell Tissue Res. 2010;341:397-404.

11. Erickson GA, Bolin SR, Landgraf JG. Viral contamination of fetal bovine serum used for tissue culture: risks and concerns. Dev Biol Stand. 1991;75:173-5.

12. Sakamoto N, Tsuji K, Muul LM, et al. Bovine apolipoprotein $\mathrm{B}-100$ is a dominant immunogen in therapeutic cell populations cultured in fetal calf serum in mice and humans. Blood. 2007;110:501-8.
13. Agata H, Watanabe N, Ishii Y, et al. Feasibility and efficacy of bone tissue engineering using human bone marrow stromal cells cultivated in serum-free conditions. Biochem Biophys Res Commun. 2009;382:353-8.

14. Chase LG, Lakshmipathy U, Solchaga LA, Rao MS, Vemuri MC. A novel serum-free medium for the expansion of human mesenchymal stem cells. Stem Cell Res Ther. 2010;1:8.

15. Lindroos B, Boucher S, Chase L, et al. Serum-free, xeno-free culture media maintain the proliferation rate and multipotentiality of adipose stem cells in vitro. Cytotherapy. 2009;11:958-72.

16. Grayson WL, Zhao F, Bunnell B, Ma T. Hypoxia enhances proliferation and tissue formation of human mesenchymal stem cells. Biochem Biophys Res Commun. 2007;358:948-53.

17. Dos Santos F, Andrade PZ, Boura JS, Abecasis MM, da Silva CL, Cabral JM. Ex vivo expansion of human mesenchymal stem cells: a more effective cell proliferation kinetics and metabolism under hypoxia. J Cell Physiol. 2010;223:27-35.

18. Sato K, Itoh T, Kato T, et al. Serum-free isolation and culture system to enhance the proliferation and bone regeneration of adipose tissue-derived mesenchymal stem cells. In Vitro Cell Dev Biol Anim. 2015;51:515-29.

19. Nakahara T, Tominaga N, Toyomura J, Tachibana T, Ide Y, Ishikawa $\mathrm{H}$. Isolation and characterization of embryonic ameloblast lineage cells derived from tooth buds of fetal miniature swine. In Vitro Cell Dev Biol Anim. 2016;52:445-53.

20. Nakahara T, Tamaki Y, Tominaga N, et al. Novel amelanotic and melanotic cell lines NM78-AM and NM78-MM derived from a human oral malignant melanoma. Hum Cell. 2010;23:15-25.

21. Tominaga $N$, Nakahara $T$, Nasu $M$, Satoh $T$. Isolation and characterization of epithelial and myogenic cells by "fishing" for the morphologically distinct cell types in rat primary periodontal ligament cultures. Differentiation. 2013;85:91-100.

22. Crapnel K, Blaesius R, Hastings A, Lennon DP, Caplan AI, Bruder SP. Growth, differentiation capacity, and function of mesenchymal stem cells expanded in serum-free medium developed via combinatorial screening. Exp Cell Res. 2013;319: 1409-18.

23. Chase LG, Yang S, Zachar V, et al. Development and characterization of a clinically compliant xeno-free culture medium in good manufacturing practice for human multipotent mesenchymal stem cells. Stem Cells Transl Med. 2012;1:750-8.

24. Hung SP, Ho JH, Shih YR, Lo T, Lee OK. Hypoxia promotes proliferation and osteogenic differentiation potentials of human mesenchymal stem cells. J Orthop Res. 2012;30:260-6.

25. Fehrer C, Brunauer R, Laschober G, et al. Reduced oxygen tension attenuates differentiation capacity of human mesenchymal stem cells and prolongs their lifespan. Aging Cell. 2007;6: 745-57.

26. Salim A, Nacamuli RP, Morgan EF, Giaccia AJ, Longaker MT. Transient changes in oxygen tension inhibit osteogenic differentiation and Runx2 expression in osteoblasts. J Biol Chem. 2004;279:40007-16.

27. Lennon DP, Edmison JM, Caplan AI. Cultivation of rat marrowderived mesenchymal stem cells in reduced oxygen tension: effects on in vitro and in vivo osteochondrogenesis. J Cell Physiol. 2001; 187:345-55.

28. Liu TM, Wu YN, Guo XM, Hui JH, Lee EH, Lim B. Effects of ectopic Nanog and Oct4 overexpression on mesenchymal stem cells. Stem Cells Dev. 2009;18:1013-22.

29. Tsai CC, Su PF, Huang YF, Yew TL, Hung SC. Oct4 and Nanog directly regulate Dnmt1 to maintain self-renewal and undifferentiated state in mesenchymal stem cells. Mol Cell. 2012;47: 169-82.

30. Markway BD, Tan GK, Brooke G, Hudson JE, Cooper-White JJ, Doran MR. Enhanced chondrogenic differentiation of human bone marrow-derived mesenchymal stem cells in low oxygen 
environment micropellet cultures. Cell Transplant. 2010;19: $29-42$.

31. Adesida AB, Mulet-Sierra A, Jomha NM. Hypoxia mediated isolation and expansion enhances the chondrogenic capacity of bone marrow mesenchymal stromal cells. Stem Cell Res Ther. 2012;3:9.

32. Wu TT, Li LF, Du R, Jiang L, Zhu YQ. Hydrogen peroxide induces apoptosis in human dental pulp cells via caspase-9 dependent pathway. J Endod. 2013;39:1151-5.

33. Wang FW, Wang Z, Zhang YM, et al. Protective effect of melatonin on bone marrow mesenchymal stem cells against hydrogen peroxide-induced apoptosis in vitro. J Cell Biochem. 2013;114:2346-55.

34. Williams CG, Malik AN, Kim TK, Manson PN, Elisseeff JH. Variable cytocompatibility of six cell lines with photoinitiators used for polymerizing hydrogels and cell encapsulation. Biomaterials. 2005;26:1211-8.
35. Fedorovich NE, Oudshoorn MH, van Geemen D, Hennink WE, Alblas J, Dhert WJ. The effect of photopolymerization on stem cells embedded in hydrogels. Biomaterials. 2009;30:344-53.

36. Kennea NL, Stratou C, Naparus A, Fisk NM, Mehmet H. Functional intrinsic and extrinsic apoptotic pathways in human fetal mesenchymal stem cells. Cell Death Differ. 2005;12:1439-41.

37. Oliver L, Hue E, Rossignol J, et al. Distinct roles of Bcl-2 and $\mathrm{Bcl}-\mathrm{Xl}$ in the apoptosis of human bone marrow mesenchymal stem cells during differentiation. PLoS One. 2011;. doi:10.1371/ journal.pone.0019820.

38. McKee MD, Nanci A. Osteopontin: an interfacial extracellular matrix protein in mineralized tissues. Connect Tissue Res. 1996;35:197-205.

39. Saito M, Iwase M, Maslan S, et al. Expression of cementumderived attachment protein in bovine tooth germ during cementogenesis. Bone. 2001;29:242-8. 